\title{
Study on Compatibility of Polymer Hydrodynamic Size and Pore Throat Size for Honggang Reservoir
}

\author{
Dan-Dan Yin, ${ }^{1,2}$ Yi-Qiang Li, ${ }^{1,2}$ Bingchun Chen, ${ }^{3}$ Hui Zhang, ${ }^{3}$ \\ Bin Liu, ${ }^{3}$ Qingle Chang, ${ }^{1,2}$ and Yanyue $\mathrm{Li}^{1,2}$ \\ ${ }^{1}$ EOR Research Institute, China University of Petroleum, Beijing 102249, China \\ ${ }^{2}$ Key Laboratory of Petroleum Engineering, China University of Petroleum, Beijing 102249, China \\ ${ }^{3}$ Exploration and Development Research Institute of Jilin Oilfield, PetroChina, Songyuan 138000, China \\ Correspondence should be addressed to Dan-Dan Yin; yindandan01@foxmail.com
}

Received 11 May 2014; Revised 23 July 2014; Accepted 23 July 2014; Published 11 August 2014

Academic Editor: Jan-Chan Huang

Copyright (C) 2014 Dan-Dan Yin et al. This is an open access article distributed under the Creative Commons Attribution License, which permits unrestricted use, distribution, and reproduction in any medium, provided the original work is properly cited.

\begin{abstract}
Long core flow experiment was conducted to study problems like excessive injection pressure and effective lag of oil wells during the polymer flooding in Honggang reservoir in Jilin oilfield. According to the changes in viscosity and hydrodynamic dimensions before and after polymer solution was injected into porous media, the compatibility of polymer hydrodynamic dimension and the pore throat size was studied in this experiment. On the basis of the median of radius $R$ of pore throats in rocks with different permeability, dynamic light scattering method (DLS) was adopted to measure the hydrodynamic size Rh of polymer solution with different molecular weights. The results state that three kinds of $1500 \mathrm{mg} / \mathrm{L}$ concentration polymer solution with $2000 \times 10^{4}, 1500 \times$ $10^{4}$, and $1000 \times 10^{4}$ molecular weight matched well with the pore throat in rocks with permeability of $300 \mathrm{mD}, 180 \mathrm{mD}$, and $75 \mathrm{mD}$ in sequence. In this case, the ratios of core pore throat radius median to the size of polymer molecular clew $R / R_{h}$ are $6.16,5.74$, and 6.04. For Honggang oil reservoir in Jilin, when that ratio ranges from 5.5 to 6.0 , the compatibility of polymer and the pore structure will be relatively better.
\end{abstract}

\section{Introduction}

Polymer flooding can improve the water-oil mobility ratio in the oil layer, effectively enlarge the swept volume, and be capable of enhancing oil recovery up to $10 \%$, and this technology has already been carried out in Daqing oilfield [1-4]. The polymers used for flooding (partially hydrolyzed polyacrylamide (HPAM)) are trackless coils consisting of one or more twined molecular chains in the solution [5], and the clew size is affected by many factors, such as the relative molecular weight of polymer, polymer concentration, and the ionic concentration of the sample water [6-8]. Theoretically, for some reservoirs, under a certain concentration, with increasing relative molecular weight of the polymer, the viscosity improves, the hydrodynamic size increases, and the control ability over fluidity gets stronger $[9,10]$. However, during the real injection process, the polymer used as oildisplacing agent will be naturally selected by the pore throat sizes as flowing through porous medium instead of entering the pores and throats in the porous mediums completely [11]. When the hydrodynamic size is way larger than the throat size of rock and under normal injection pressure, the polymer molecules will accumulate in the pipeline or at the core entrance and only a small amount of relative small molecular clews can enter the core [12]. Even though under some external forces, molecular clews with larger size may manage to enter the cores, the molecular structure will be damaged, and the displacement will be ineffective [13]; in addition, the migration distances for the polymer molecules entering the cores will be relatively short, which also make it difficult to generate effective displacement ability. Taking effect inconspicuously in oil wells is a typical issue at the mine field, and some objective intervals will also have some issues like pressure skyrocketing and failing to inhale, both of which will weaken the injectability. So how to improve the effect of polymer flooding and further enlarge the swept volume so as 
TABLE 1: Water quality parameters of the on-site injection water from Honggang oilfield in Jilin.

\begin{tabular}{lcccccc}
\hline Ion & $\mathrm{K}^{+}, \mathrm{Na}^{+}$ & $\mathrm{Mg}^{2+}$ & $\mathrm{Ca}^{2+}$ & $\mathrm{Cl}^{-}$ & $\mathrm{SO}_{4}{ }^{2-}$ & $\mathrm{HCO}_{3}{ }^{-}$ \\
\hline $\begin{array}{l}\text { Concentration } \\
(\mathrm{mg} / \mathrm{L})\end{array}$ & 162.5 & 36.7 & 87.8 & 127.6 & 164.6 & 454.2 \\
\hline
\end{tabular}

to enhance the reserve utilization degree has been a research hotspot [14]. Therefore, it is of paramount importance for formulating the scheme and developing polymer flooding technology to study the matching relation between polymer and reservoirs with different permeability through analyzing the compatibility of hydrodynamic size and pore throat size. Polymer solution flooding experiment was conducted with $10 \mathrm{~cm}$ long core by predecessors [11], and the compatibility of polymer hydrodynamic dimension and the pore throat size was studied just according to the core inlet pressure. However, long cores were not used to test pressure distribution in the core yet. Besides, the changes of viscosity and the molecular size of the effluent were not tested in other experiments.

In this paper, long cores with different permeability and 4 kinds of polymer with different molecular weight in $\mathrm{X}$ Block of Honggang reservoir located in Jilin oilfield were taken as the samples. The core flow test was used to detect the propagation rule of pressure during polymer injection process and judge the migration features of polymer. The changes in the viscosity of polymer solution and the clew sizes pre- and postcore injection were tested, so as to study the compatibility of hydrodynamic size and pore throat size.

\section{Experiment}

2.1. Experimental Materials and Apparatus. The polymers utilized were 4 kinds of HPAM named after p1, p2, p3, and $\mathrm{p} 4$ in this paper with different relative molecular weights of $800 \times 10^{4}, 1500 \times 10^{4}, 2000 \times 10^{4}$, and $2500 \times 10^{4}$, respectively, and the effective solid content was $80 \%$. The water used for preparing liquid was taken from the injection water on site of Honggang reservoir in Jilin oilfield with $1033.4 \mathrm{mg} / \mathrm{L}$ salinity. The ion composition and concentration are exhibited in Table 1. Water for preparing the fluid was prepared by filtering sample water on $0.22 \mu \mathrm{m}$ membranes and storing. The formation fracture pressure of target Block X is $52 \mathrm{MPa}$.

The homogeneous long core used in the experiment was entirely pressed by outcrops with different radiuses. First, the outcrops were crushed into particles, and then particles were pressed into the cylindrical core with inorganic cementing agent under certain pressure. Their pore sizes are close to each other according to the mercury injection experiment. Compared with the quartz sand used for making normal core, the particle shape and surface properties of the outcrop sand are more similar to a real core because they have the same mineral composition. The core had $2.5 \mathrm{~cm}$ radius and $100 \mathrm{~cm}$ length. According to the reservoir permeability distribution of X Block in Jilin Honggang oilfield, representative cores were chosen with $300 \mathrm{mD}, 180 \mathrm{mD}$, and $75 \mathrm{mD}$ air permeability, $148 \mathrm{mD}, 87 \mathrm{mD}$, and $30 \mathrm{mD}$ water permeability, and $23.4 \%, 22.11 \%$, and $20.32 \%$ porosity, and their pore throat radius medians were $3.14 \mu \mathrm{m}, 2.13 \mu \mathrm{m}$, and $1.51 \mu \mathrm{m}$, respectively, according to the mercury injection test.

Main experimental facilities are as follows: self-designed long core physical simulation equipment (Figure 1) including a long core holding unit with surveying points (the surveying points in the middle divided the model into two equal parts), incubator, pressure sensor with high precision, data acquisition system, ISCO pump with constant velocity and pressure, HAAKE Rotational Rheometer, and microburette.

2.2. Experimental Method. Prepare the polymers with different molecular weights into a mother solution with $5000 \mathrm{mg} / \mathrm{L}$ concentration by using the on-site injection water and leave it to stand for $4 \mathrm{~h}$ and then dilute it into $1500 \mathrm{mg} / \mathrm{L}$ target solution. After that, shift 5 of warring blender shear apparatus was used to simulate the shearing action of the shot hole for $10 \mathrm{~s}$ before storage.

2.2.1. Determination of Viscosity. The viscosities of the polymer solutions before and after injection were determined at a shear rate of $7.34 \mathrm{~s}^{-1}$ with HAAKE Rotational Rheometer6000 at $55^{\circ} \mathrm{C}$.

2.2.2. Determination of Polymer Molecular Clew Size. American Brookhaven BI-200SM wide angle dynamic/static light scattering with $523 \mathrm{~nm}$ wavelength was adopted, and the concentration of polymer solution was $100 \mathrm{mg} / \mathrm{L}$. DLS method has wide measurement range, high measurement speed, and less sample amount.

\subsubsection{Mobility Test}

(1) Vacuumize the core and saturate the formation water, measure the core permeability by water, and calculate the porosity.

(2) Conduct water flooding and polymer flooding under $55^{\circ} \mathrm{C}$, record the injection pressure, collect the produced liquid, and measure relevant parameters.

(3) Conduct water flooding under $55^{\circ} \mathrm{C}$ for a second time, record the injection pressure, and measure relevant parameters.

Referring to the responding time of polymer flooding in Daqing oilfield, the linear velocity of effective flow for the fluid flowing in the stratum was figured out to be $1 \mathrm{~m} / \mathrm{d}$. Therefore, 3-5 PV polymer solution was injected continuously with an injection flow of $0.1 \mathrm{~mL} / \mathrm{min}$ until a stabilized situation was achieved.

\section{Experimental Results and Discussion}

3.1. Injection Characters of Polymer Solution. The propagation performance of polymers propagating in the porous medium was evaluated primarily, that is to say, whether there is retention phenomenon during the injection process. The propagation rules of pressure were evaluated principally. If 


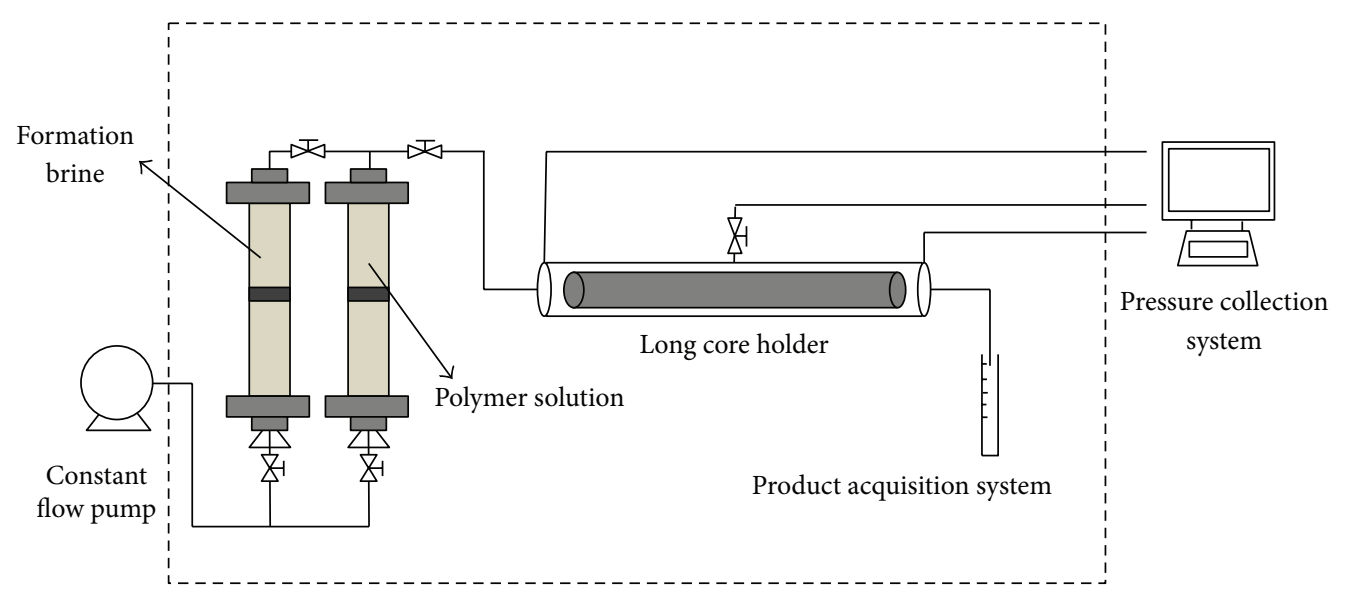

FIGURE 1: Flow chart of injection experiment.

the polymer solution has preferable propagation performance in the porous medium, then no block will emerge and the pressure will drop equally throughout homogeneous cores. Moreover, the resistance coefficients of the front half and latter half of the core will be close. Resistance coefficient (RF) is the ratio between water and polymer solution mobility:

$$
\mathrm{RF}=\frac{\lambda_{w}}{\lambda_{\mathrm{p}}}=\frac{\mathrm{K}_{w} / \mu_{w}}{\mathrm{~K}_{\mathrm{p}} / \mu_{\mathrm{p}}} .
$$

In the equation, RF is the resistance coefficient (a nondimensional parameter); $\lambda_{w}$ is the water mobility; $\lambda_{\mathrm{p}}$ is the polymer solution mobility; $\mathrm{K}_{w}$ is the water phase permeability, in $\mathrm{mD} ; \mathrm{K}_{\mathrm{p}}$ is the polymer solution permeability, in $\mathrm{mD} ; \mu_{w}$ is the viscosity of water, in $\mathrm{mPa} \cdot \mathrm{s} ; \mu_{\mathrm{p}}$ is the viscosity of polymer solution, in $\mathrm{mPa} \cdot \mathrm{s}$.

When the hydrodynamic size of the polymer is larger than the throat size of rock, the polymer molecules will accumulate at the core entrance or travel short distances in the core, so the pressure and resistance coefficients in the front and back long core sections are very different. When the hydrodynamic size of the polymer has a very good compatibility with the throat size of rock, the polymer molecules will travel smoothly in the core and are distributed evenly throughout the core. So the pressure and resistance coefficients in the front and back long core sections are much closed. Figure 2 shows the pressure variation with the change in injection volume when 4 kinds of polymer solutions were injected into 3 kinds of cores with different permeability. A surveying point on the experimental model divided the model into two parts (PX-1 and PX-2 are the pressure at the core entrance and the middle part, resp.). When the pressure on the surveying points goes up, it indicates that the polymer solution has migrated to this part. During the process of chemical agent injection, it is the pressure around the entrance that rose first, and the pressure at the middle increased along with the chemical agent migration. Four kinds of polymer solution with different molecular weights were injected into cores with $300 \mathrm{mD}, 180 \mathrm{mD}$, and $75 \mathrm{mD}$ permeability, and their variation tendencies are shown in Figures 2(a), 2(b), and 2(c), respectively.
Figure 2(a) shows the pressure variation with injection volume by injecting solutions prepared by p2, p3, and p4 polymers into a core with $300 \mathrm{mD}$ permeability. As shown in the figure, when a small amount of $\mathrm{p} 4$ polymer solution (25 million molecular weight) was injected, the pressure at the entrance surged while pressure in the middle showed no obvious variation, the differential pressure between the front and back sections was $7.12 \mathrm{MPa}$, and the resistance coefficients of the two sections were 389.3 and 3.7, respectively. This meant that the polymer solution mainly accumulated at the core entrance, and only when the injection volume reached 2.0 PV, pressure in the middle would finally increase slightly. The injected polymer molecules also accumulated on the front part of the core and failed to achieve remote migration. The phenomenon in return explained on-site injection well pressure build up and injection failure issues at the oilfield. Pressure unable to delivery efficiently will lead to injection pressure build up or even fracture at the stratum. Moreover, during the injection process of $\mathrm{p} 4$ solution, the pressure was always changing, which meant that the polymer molecular clews were oversized and mismatched with the size of formation pores. The displacement agent of $\mathrm{p} 3$ solution was prepared by polymers with 20 million molecular weight, the pressure increased gradually from the core entrance (P3-1) to the middle part (P3-2) with a steady rising velocity, the differential pressure between the entrance and the middle part was $2.13 \mathrm{MPa}$, and the resistance coefficients of the front and back sections were 106.5 and 105, respectively. This reflected the steady migration process of chemical agent in the model and preferable compatibility of polymer molecular clew size and the pore size. During the process of p2 solution injection, the pressure increased gradually from the core exit (P2-1) to the entrance (P2-2) with steady rising velocity, which further reflects the stable migration process of chemical slug in the model. However, due to the low injection pressure in the injection system, the differential pressure was 1.03 $\mathrm{MPa}$, and the resistance coefficients of the front and back parts were 53 and 46, respectively. The excessive velocity of the injection system in the large pores led to a failure of controlling the fluidity, which means that polymer molecular clew was undersized and mismatched with the pore size. 


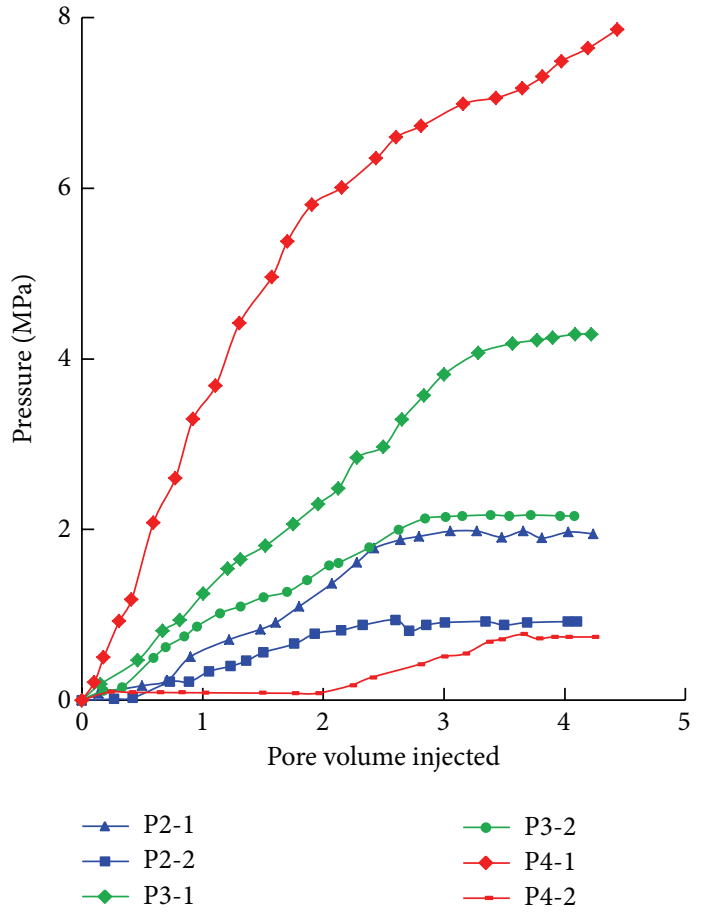

(a)

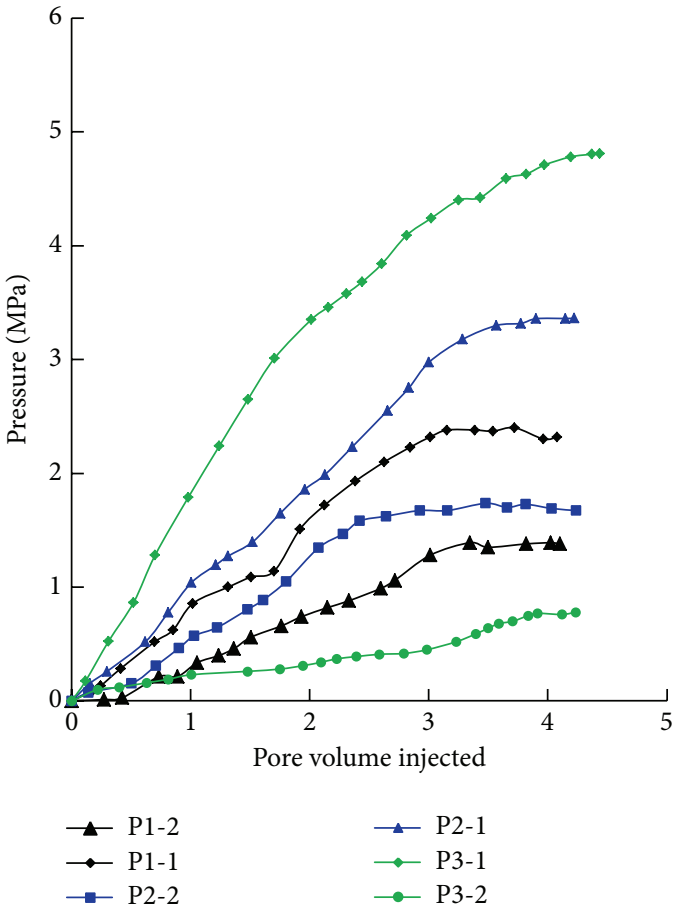

(b)

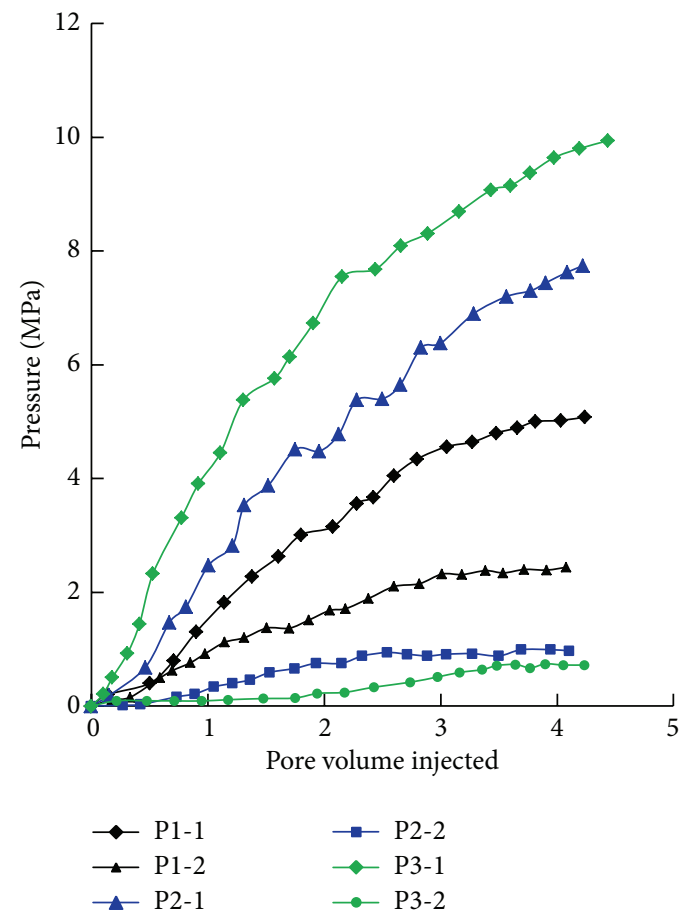

(c)

FIGURE 2: Injection pressures variation with injection volume of different polymer solutions ((a) Kg: 300 mD; (b) Kg: 180 mD; (c) Kg: 75 mD).

Figure 2(b) is a diagram of the pressure variation with the injection volume as injecting polymers $\mathrm{p} 1, \mathrm{p} 2$, and $\mathrm{p} 3$ into a core with $180 \mathrm{mD}$ permeability. The pressure of solution prepared by $\mathrm{p} 3$ increased at the entrance (P3-1), while there was no change in the middle (P3-2), the differential pressure was $3.3 \mathrm{MPa}$, and the resistance coefficients of the front and back sections were 201 and 39, respectively. This means that the polymer solution mainly accumulated at the core entrance, and the oversized polymer molecular clew in the solution prepared by $\mathrm{p} 3$ mismatched with the pore 
TABLE 2: Viscosity and its loss rate in the produced liquid with 4 kinds.

\begin{tabular}{|c|c|c|c|c|c|c|c|c|}
\hline \multirow{2}{*}{$\mathrm{Kg}, \mathrm{mD}$} & \multicolumn{2}{|c|}{ pl } & \multicolumn{2}{|l|}{ p2 } & \multicolumn{2}{|c|}{ p3 } & \multicolumn{2}{|c|}{$\mathrm{p} 4$} \\
\hline & Viscosity, mPa.s & Loss rate, $\%$ & Viscosity, $\mathrm{mPa} \cdot \mathrm{s}$ & Loss rate, $\%$ & Viscosity, $\mathrm{mPa} \cdot \mathrm{s}$ & Loss rate, $\%$ & Viscosity, mPa.s & Loss rate, $\%$ \\
\hline 300 & 12.36 & 4.31 & 21.13 & 5.95 & 41.95 & 8.26 & 51.94 & 31.12 \\
\hline 180 & 11.96 & 7.45 & 20.27 & 9.77 & 33.23 & 27.32 & 34.89 & 53.73 \\
\hline 75 & 11.77 & 9.16 & 13.76 & 38.77 & 22.85 & 50.03 & 21.48 & 71.51 \\
\hline
\end{tabular}

The viscosities of the four kinds of polymer solution with $1500 \mathrm{mg} / \mathrm{L}$ concentration were $75.40 \mathrm{mPa} \cdot \mathrm{s}, 45.73 \mathrm{mPa} \cdot \mathrm{s}, 22.47 \mathrm{mPa} \cdot \mathrm{s}$, and $12.92 \mathrm{mPa} \cdot \mathrm{s}$ in sequence before injection.

TABLE 3: Changes in the hydrodynamic dimensions of the produced liquid from polymer solution.

\begin{tabular}{lcccccccc}
\hline $\begin{array}{l}\text { Kg } \\
\mathrm{mD}\end{array}$ & $R_{h}, \mu \mathrm{m}$ & Loss rate, $\%$ & $R_{h}, \mu \mathrm{m}$ & Loss rate, $\%$ & $R_{h}, \mu \mathrm{m}$ & Loss rate, $\%$ & $R_{h}, \mu \mathrm{m}$ & Loss rate, \% \\
\hline 300 & 0.24 & 4.0 & 0.34 & 5.6 & 0.46 & 9.8 & 0.52 & 18.8 \\
180 & 0.23 & 8.0 & 0.32 & 11.1 & 0.42 & 17.6 & 0.45 \\
75 & 0.22 & 12.0 & 0.28 & 22.2 & 0.34 & 33.3 & 0.36 & 43.8 \\
\hline
\end{tabular}

The hydrodynamic dimensions of 4 kinds of polymer solutions p1, p2, p3, and p4 with $100 \mathrm{mg} / \mathrm{L}$ concentration are $0.25 \mu \mathrm{m}, 0.36 \mu \mathrm{m} 0.51 \mu \mathrm{m}$, and $0.64 \mu \mathrm{m}$, respectively (the order was ranked by their molecular weights).

size in a stratum with $180 \mathrm{mD}$ permeability. The pressure variation tendency of the solution prepared by $\mathrm{p} 2$ shows was approximately linear increase from the core entrance to the middle part, the differential pressure between the surveying points at the entrance and in the middle was 1.69 $\mathrm{MPa}$, and the resistance coefficients of the front and back sections were 84.5 and 83 , respectively. This means that the chemical agent migrated steadily in the core and implies a good compatibility of polymer molecular clew size and pore size. Figure 2(c) shows the pressure variation with the injection volume as $\mathrm{p} 1, \mathrm{p} 2$, and $\mathrm{p} 3$ polymers were injected into a core with $75 \mathrm{mD}$ permeability. The pressure variation of solution prepared by $\mathrm{p} 1$ increased uniformly from the core entrance to the middle part, the differential pressure between the entrance and the middle part was $2.56 \mathrm{MPa}$, and the resistance coefficients of the front and back sections were 128 and 122, respectively, which illustrated that the polymer molecular clew size matched well with the pore size.

3.2. Changes in Polymer Solution Viscosity. Table 2 shows the viscosity changes in produced liquid after 4 kinds of polymer solution were injected into 3 kinds of cores with different permeability.

The polymer viscosity loss was mainly due to the shearing and absorption effect, and several conclusions are suggested by the data: for cores with the same permeability, the viscosity loss rate of the produced liquid increased with the polymer molecular weight. This means that with increasing molecular weight, the length of polymer molecular chain after hydration increased and the polymer became easier to be broken under stress during the migration process in the pores, leading to an increase in the viscosity loss rate. Under the same polymer molecular weight conditions, smaller core permeability means smaller pore size, more complex structure, which will result in stronger shearing effect on polymer, larger specific surface area of core, more severe absorption effect on polymers, and, as a consequence, a serious viscosity loss of the polymer solution.

Combined with the flow test in the cores and conducting some further analysis, p1, p2, and p3 matched well with the cores with $300 \mathrm{mD}, 180 \mathrm{mD}$, and $75 \mathrm{mD}$ permeability, and these three kinds of polymer solutions had little viscosity loss rate in the cores with corresponding permeability and stable pressure. To further verify their matching relations, microanalysis is required to measure changes in hydrodynamic dimensions.

3.3. Changes in the Polymer Hydrodynamic Dimensions. Four kinds of polymer solution with different molecular weight and $1500 \mathrm{mg} / \mathrm{L}$ concentration were injected into cores with $300 \mathrm{mD}, 180 \mathrm{mD}$, and $75 \mathrm{mD}$ permeability at $0.1 \mathrm{~mL} / \mathrm{min}$ velocity until the pressure was stable, after which the hydrodynamic dimensions of produced liquid at the exit were measured, and both the polymer solution and produced liquid were diluted to $100 \mathrm{mg} / \mathrm{L}$. The polymer molecular clew sizes and light scattering distribution results are reported in Table 3.

As shown in Figure 3, the average hydrodynamic dimension $R_{h}$ of the polymer solution with $800 \times 10^{4}, 1500 \times 10^{4}$, $2000 \times 10^{4}$, and $2500 \times 10^{4}$ molecular weights before injection were $0.25 \mu \mathrm{m}, 0.36 \mu \mathrm{m}, 0.51 \mu \mathrm{m}$, and $0.64 \mu \mathrm{m}$, respectively, and the peak values are relatively concentrated. After flowing over the core, the $R_{h}$ of polymer solution decreased, and, for the same core permeability conditions, a larger polymer molecular weight means greater drop in hydrodynamic dimension; for solutions with the same polymer molecular weight, lower permeability means greater drop degree of the hydrodynamic dimension, which is mainly due to the low permeability and small pore size. This makes a molecular clew with relatively larger size unable to pass through the pores with its molecular structure undamaged. After passing through cores with different permeability, the peak value of polymer $R_{h}$ moved towards left and the proportion of 


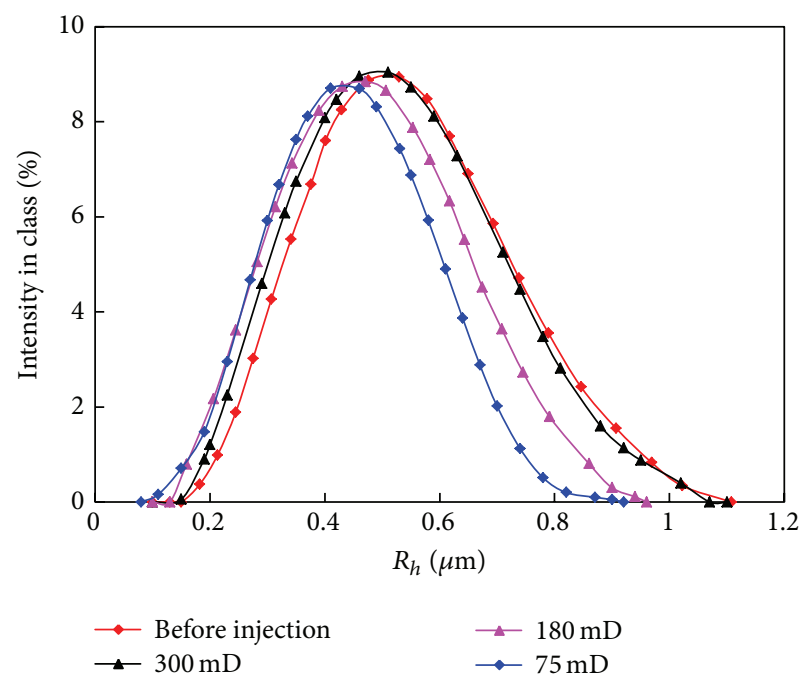

(a) $M=1000 \times 10^{4}$

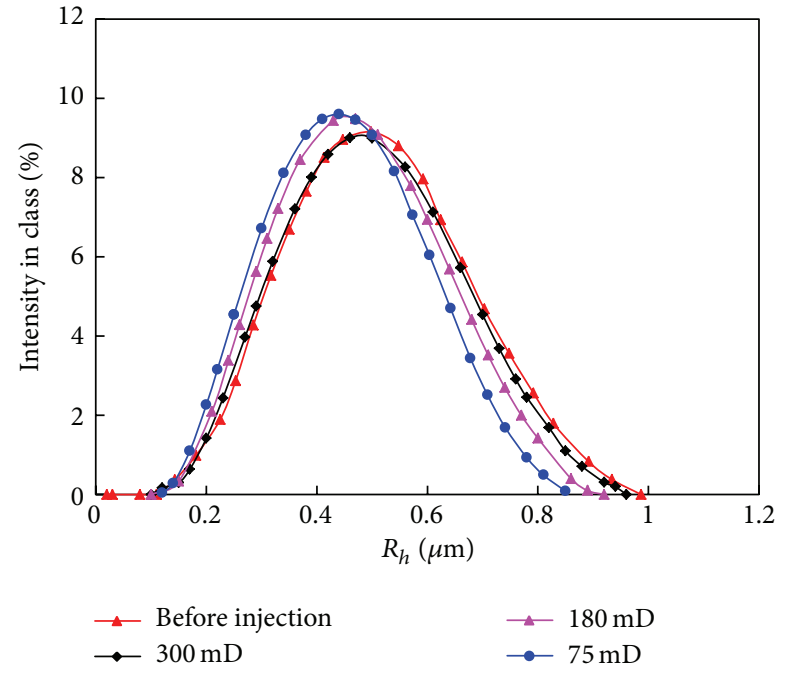

(b) $M=1500 \times 10^{4}$

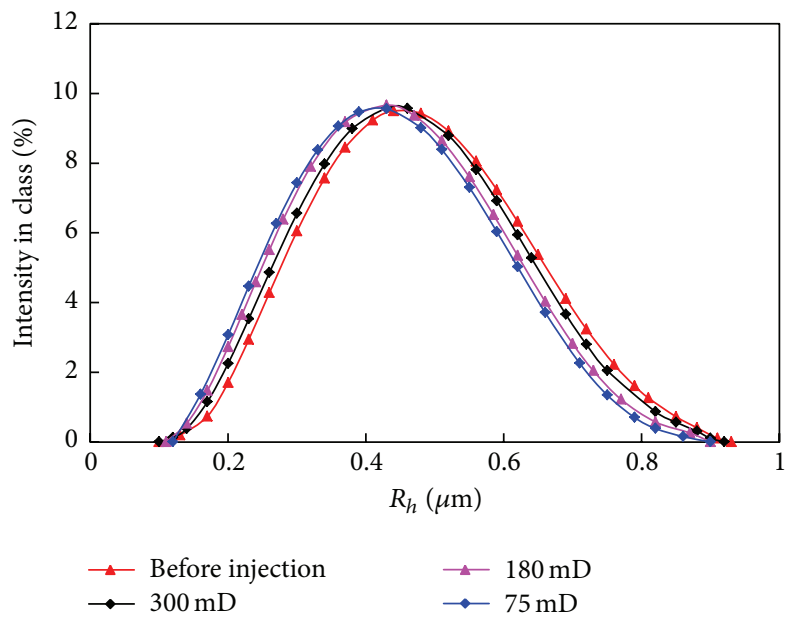

(c) $M=2000 \times 10^{4}$

FIGURE 3: $R_{h}$ distribution of polymer solution with different relative molecular mass before and after injection of different permeability cores.

polymers with relatively smaller hydrodynamic dimensions increased. In contrast, the proportion of polymers with relatively larger $R_{h}$ decreased and the phenomenon can be explained in this way: when the polymers passed through the pores and throats in the core, the quantity of polymer with larger $R_{h}$ decreased with decreasing core permeability, and that was consistent with the conclusion about viscosity loss rate.

3.4. Compatibility of Polymer Molecular Clew Size and Pore Throat Size. As different researchers defined differently the pore radius, the water used for preparing polymer solution had different salinity, and, for different reservoir conditions, the specific values $R / R_{h}$ for representative polymer and core compatibility were hard to determine.

In this paper, the reservoir conditions in Honggang oilfield were used, and the polymer hydrodynamic size and the pore throat radius were unified at their average values. Table 4 shows the relevant data.
TABle 4: Calculation results of the specific value of pore throat radius median to average hydrodynamic dimension.

\begin{tabular}{lcccc}
\hline \multicolumn{2}{c}{ Polymer parameters } & \multicolumn{2}{c}{ Core parameters } & $R / R_{h}$ \\
$M$ & $R_{h} / \mu \mathrm{m}$ & $\mathrm{Kg} / \mathrm{mD}$ & $R / \mu \mathrm{m}$ & \\
\hline $2000 \times 10^{4}$ & 0.51 & 300 & 3.14 & 6.16 \\
$1500 \times 10^{4}$ & 0.38 & 180 & 2.18 & 5.74 \\
$1000 \times 10^{4}$ & 0.25 & 75 & 1.51 & 6.04 \\
\hline
\end{tabular}

To avoid polymer retention in the pore throats in the stratum of Honggang reservoir, the core permeability, polymer concentration, relative molecular weight, and the chemical composition of the solvent water were taken into consideration. Generally, $R / R_{h}$ ranges from 5.5 to 6.0 . For stratum with $300 \mathrm{mD}, 180 \mathrm{mD}$, and $75 \mathrm{mD}$ permeability, polymers with relative molecular weight lower than $2000 \times 10^{4}, 1500 \times 10^{4}$, and $1000 \times 10^{4}$ are recommended, and if the shearing and degradation effect during preparation and injection processes 
are taken into consideration, the relative molecular weights of polymers can still be increased modestly.

\section{Conclusions}

(1) When the polymer solution was injected into cores with different permeability, the injection pressure had varying degrees of increase. Three kinds of polymers with $800 \times 10^{4}, 1500 \times 10^{4}$, and $2000 \times 10^{4}$ molecular weights matched well with the pore throats in cores with permeability of $300 \mathrm{mD}, 180 \mathrm{mD}$, and $75 \mathrm{mD}$, respectively. They showed preferable injectability and no block at the injection end and propagated uniformly in the cores.

(2) For Honggang reservoir in Jilin oilfield, when the specific value $R / R_{h}$ (pore throat radius median/polymer clew size) is among 5.5-6.0, the matching relation between polymer and the pore structure of cores is preferable and can better suit the pore throat structure in the reservoirs.

(3) During the practical application of polymer flooding at Honggang oilfield in Jilin, for stratum with $300 \mathrm{mD}, 180 \mathrm{mD}$, and $75 \mathrm{mD}$ permeability, polymers with $2000 \times 10^{4}, 1500 \times 10^{4}$, and $1000 \times 10^{4}$ molecular weights were recommended, and if the shearing and degradation effect during preparation and injection processes are taken into consideration, the relative molecular weights of polymers can still be increased modestly.

\section{Conflict of Interests}

The authors declare no conflicts of interests regarding the publication of this paper.

\section{Acknowledgments}

The authors would like to express their appreciation for the financial support received from National Natural Science Foundation (51074172) and EOR Institute of China University of Petroleum for permission to publish this paper.

\section{References}

[1] D.-M. Wang, J.-C. Cheng, J.-Z. Wu, and G. Wang, "Application of polymer flooding technology in Daqing Oilfield," Acta Petrolei Sinica, vol. 26, no. 1, pp. 74-78, 2005.

[2] C. Jiecheng, S. Xinguang, W. Yan, J. Luo, W. Lei, and X. Qing, "Cases studies on polymer flooding for poor reservoirs in daqing oilfield," in Proceedings of the Asia Pacific Oil and Gas Conference and Exhibition, 2007.

[3] N. Jin-gang, "Practices and understanding of polymer flooding enhanced oil recovery technique in Daqing oilfield," Petroleum Geology Oilfield Development in Daqing, vol. 5, article 024, 2004.

[4] D. Wang, H. Dong, C. Lv, X. Fu, and J. Nie, "Review of practical experience of polymer flooding at Daqing," SPE Reservoir Evaluation \& Engineering, vol. 12, no. 3, pp. 470-476, 2009.
[5] L. Wenli, M. Desheng, N. Xiaobin et al., "Study on matching relation between polymer molecular size and pore size for conglomerate reservoir," in Proceedings of the International Petroleum Technology Conference Challenging Technology and Economic Limits to Meet the Global Energy Demand (IPTC '13), pp. 1778-1786, March 2013.

[6] P. Chen, Z. Shao, and Y. Liu, "Method for determining relative molecular mass of polymer in medium-low permeable reservoirs," Petroleum Geology \& Oilfield Development in Daqing, vol. 3, article 040, 2005.

[7] M. Li, Z. Liu, X. Song, B. Ma, and W. Zhang, "Effect of metal ions on the viscosity of polyacrylamide solution and the mechanism of viscosity degradation," Journal of Fuel Chemistry and Technology, vol. 40, no. 1, pp. 43-47, 2012.

[8] Z. Xiutai, W. Zengbao, and Q. Guangmin, "Study on influence factors of the initial viscosity of HPAM solution," Chemical Engineering of Oil \& Gas, vol. 3, article 15, 2009.

[9] J. Cheng, D. Wang, and J. Wu, "Molecular weight optimization for polymer flooding," Acta Petrolei Sinica, vol. 21, pp. 102-106, 2000.

[10] S. G. Goodyear, J. D. Johnston, T. A. Lawless, and C. L. Woods, "Measurement of polymer retention levels representative of the formation," in Proceedings of the SPE/DOE Enhanced Oil Recovery Symposium, 1992.

[11] L. Wenli, M. Desheng, L. Meiqin, L. Gang, N. Xiaobin, and L. Qingxia, "Matching relation between HPAM polymer DQ3500 and pores of reservoir rock," Procedia Engineering, vol. 18, pp. 261-270, 2011.

[12] T. Lotsch, T. Muller, and G. Pusch, "The effect of inaccessible pore volume on polymer coreflood experiment," in Proceedings of the SPE Oilfield and Geothermal Chemistry Symposium, 1985.

[13] Q. He, T. Young, G. P. Willhite, and D. W. Green, "Measurement of molecular weight distribution of polyacrylamides in core effluents," SPE Reservoir Engineering, vol. 5, no. 3, pp. 333-338, 1990.

[14] D. G. Hatzignatiou, U. L. Norris, and A. Stavland, "Core-scale simulation of polymer flow through porous media," Journal of Petroleum Science and Engineering, vol. 108, pp. 137-150, 2013. 

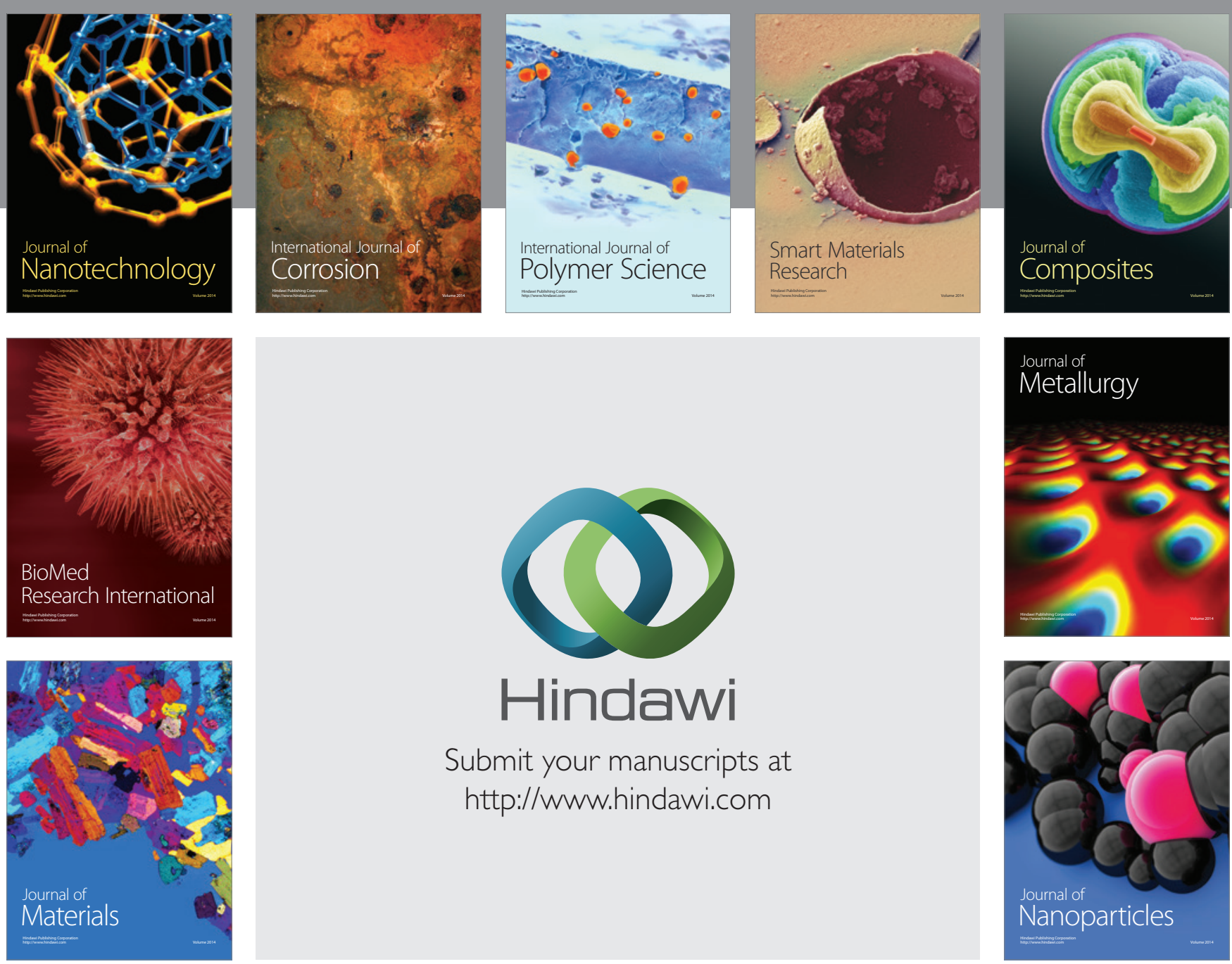

Submit your manuscripts at http://www.hindawi.com
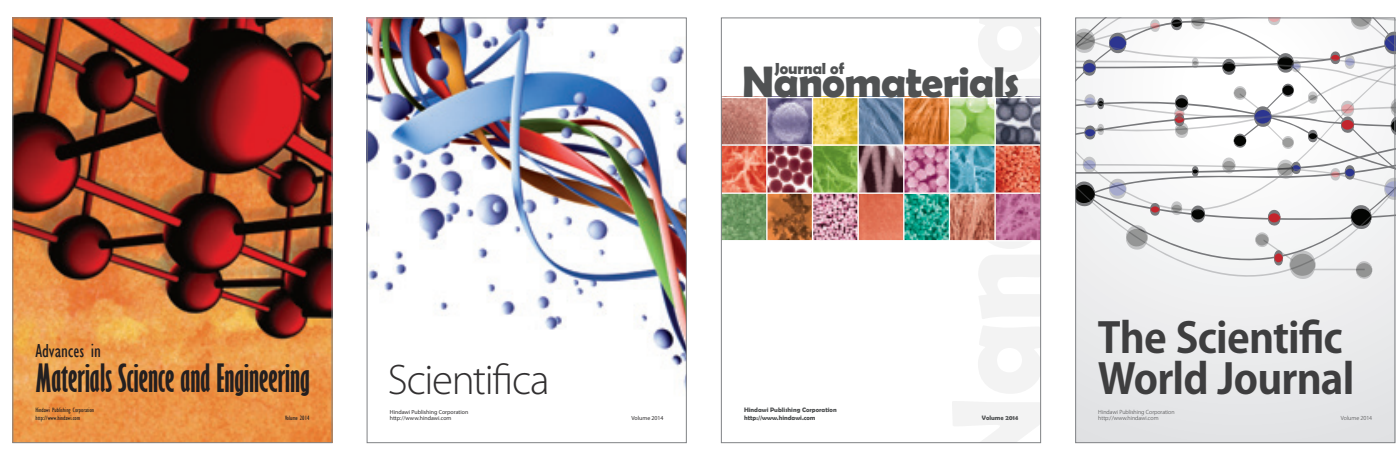

\section{The Scientific World Journal}
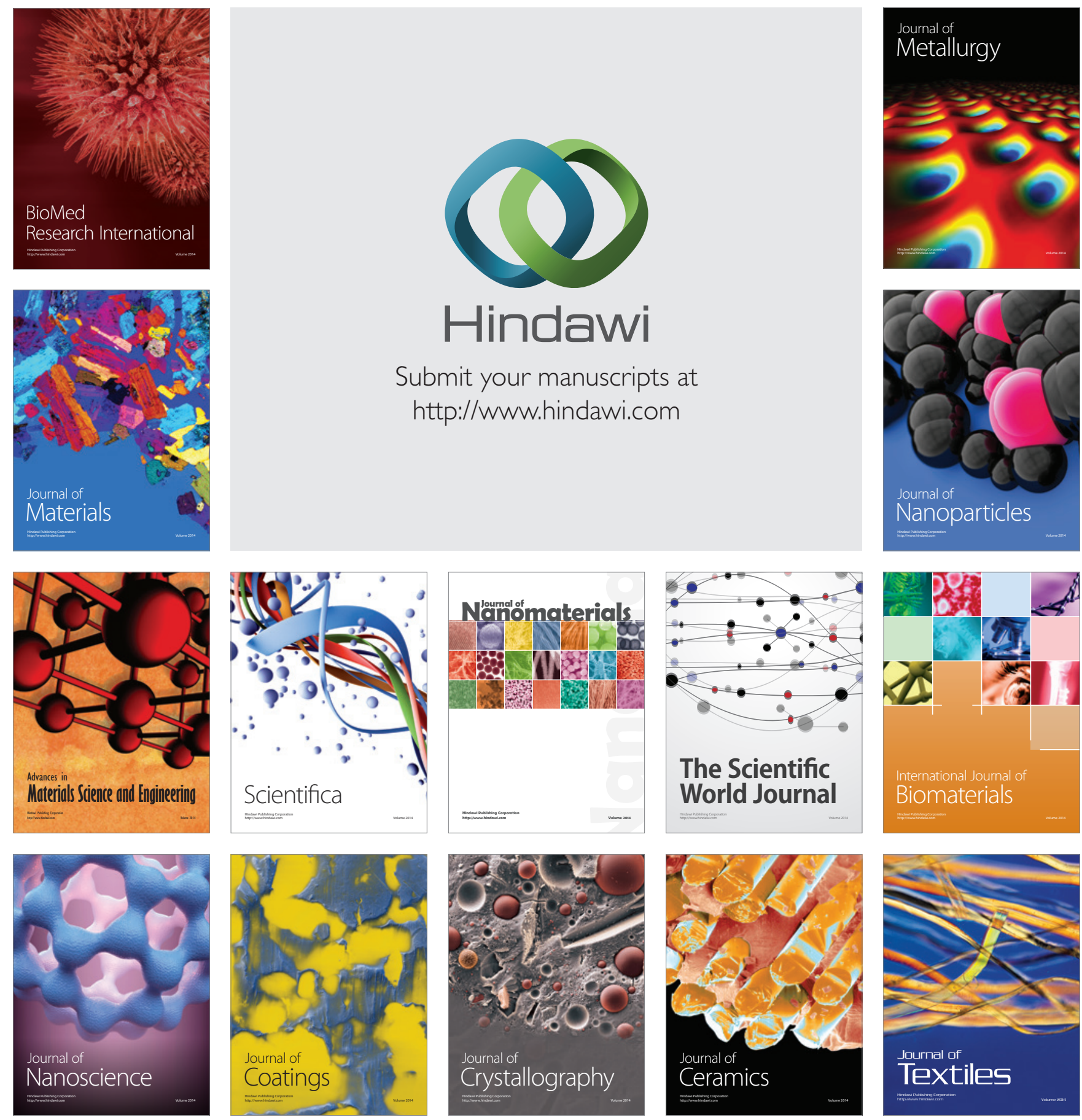